\title{
Zur Berechnung der Beobachtungen mit Bunsen's Eiscalorimeter;
}

\author{
von Demselben.
}

Bekanntlich läfst sich nie ganz verhüten, dafs durch die Aufsenwãnde des Calorimeters etwas Wărme ein- oder ausdringt, dafs also auch unabhängig von der Einführung des erwärmten Körpers etwas Quecksilber eingesogen wird oder ausfliefst. Diese positive oder negative, unabhängig von der Einführung eines erwärmten Körpers eingesogene Quecksilbermenge soll als secundär eingosogen bezeichnet werden. Sie kann angenähert der Zeit $\mathbf{t}$ proportional, also gleich a + bt gesetzt werden; gröfsere Genauigkeit werden wir erhalten, wenn wir sie gleich $a+b t+c t^{a}$ setzen. a, b und $c$ sind Constanten. Wir wollen nun annehmen, dafs durch den in's Calorimeter geworfenen Körper das Gesetz, nach welchem die secundär eingesogene Quecksilbermenge von der Zeit abhüngt, also der Werth der Constanten $b$ und $c$ gar nicht verändert wirl. Die factisch eingesogene Quecksilbermenge wird sich also jetzt folgendermafsen verhalten. Vor dem Hineinwerfen des Körpers ist sie gleich $a+b t+c^{2}$; dann bewirkı auch die vom hineingeworfenen Körper abgegebene Wärme ein Einsaugen von Quecksilber; sobald aber dieser Körper alle Wärme abgegeben hat, ist die eingesogene Quecksilbermenge wieder durch dasselle Gesetz bestimmt; nur hat die Constante a einen anderen Werth, sie ist nämlich um diejenige Quecksilbermenge $Q$ gewachsen, welche durch die vom Körper abgegebene Wärmemenge eingesogen wurde. Es ist also jetzt die eingesogene Quecksilbermenge durch $\alpha+b t+$ ct $^{2}$ gegeben, wo $b$ und $c$ dieselben Werthe baben wie frìher, $\alpha-a$ aber gleich $Q$, also der eigentich zu bestinmenden Un- 
bekannten ist *). Wir bezeichnen den beliebigen Zeitanfang mit Null und zählen alle Zeiten yon dort angefangen. $Z u$ den Zeiten $t_{1}, t_{2} \ldots t_{k}$, wo der Körper noch nicht hineingeworfen war, sollen die Beobachtungen als eingesogene Quecksilbermengen $q_{1}, q_{2} \ldots q_{k}$ ergeben, während nach der Formel die wirklich eingesogenen Quecksilbermengen a $+b t_{1}+c_{2}{ }^{1}$, $a+b t_{2}+c t_{2}{ }^{2}$ u. s. $w$. sind, so dafs der Fehler der ersten Beobachlung a $+\mathrm{bt}_{1}+\mathrm{ct}_{\mathbf{1}}{ }^{2}-\mathrm{q}_{\mathbf{1}}$ beträgt. $\mathrm{Zu}$ den Zeiten $t_{k+1}, t_{k+2} \ldots t_{k+1}$, wo die Wärmeabgabe des hineingeworfenen Körpers vollendet gewesen sein soll, sollen die Btobachtungen für die eingesogene Quecksilbermenge die Werthe $q_{k+1}, q_{k+2} \ldots q_{k+1}$ ergeben haben, deren wahren Werthe nach der Formel $a+b t_{k+1}+c_{k+1}{ }^{2}$ u. s. w. sind, Nach den Principien der Methode der kleinsten Quadrate mufs die Summe der Fehlerquadrate ein Minimurn sein. Bezeichnen wir also mit $\boldsymbol{\Sigma}_{\mathrm{k}}$ eine Summe über alle Werthe der Indices von 1 bis $k$, mit $\Sigma_{1}$ eine solche uber alle $W$ erthe von $k+1$ bis 1 und mit $\Sigma_{k l}$ eine solche von 1 bis $k+1$, so dafs $z$. B. $\boldsymbol{\Sigma}_{\mathrm{k}} \mathrm{t}^{2}=\mathrm{t}_{1}{ }^{2}+\mathrm{t}_{\mathrm{z}}{ }^{2}+\ldots \mathrm{t}_{\mathrm{k}}{ }^{2}, \boldsymbol{\Sigma}_{1} \mathrm{t}^{2}=\mathrm{t}_{\mathrm{k}+1^{2}}+\mathrm{t}_{\mathrm{k}+\mathrm{z}^{2}}+\ldots+\mathrm{t}_{\mathrm{k}+\mathrm{l}^{2}}$ u. s. w. ist, so können wir die Gröfse, welche ein Minimum werden soll, folgendermafsen schreiben :

$$
\Sigma_{\mathrm{k}}\left(\mathrm{a}+\mathrm{bt}+\mathrm{ct} \mathrm{t}^{2}-\mathrm{q}\right)^{2}+\Sigma_{\mathrm{l}}\left(\alpha+\mathrm{bt}+\mathrm{ct}^{2}-q\right)^{2}
$$

*) Darüber, wann der hineingeworfene Körper seine ganze Wärme abgegeben hat, vermögen obige Betrachtungen nichts zu lehren und mufs diefs aus anderen Wahrscheinlichkeitggründen erschlossen worden. Würde man eine einfache Hypothese über das Gesetz dieser Whrmeabgabe machen, was wohl für den späteren Verlauf derselben erlaubt sein dürfte, so könnte man aus Beobachtungen zelbst ihr Ende berechnen, respoctive die kleine Wärmeabgabe corrigiren, dio noch zu eiuer Zeit stattfindet, wo die eingesogene Quecksilbormenge schon wieder nabo der Zeit proportional ist. Hiarauf sowis auf die Falle, wo das Calorimeter zur Messung von Verbrennungswirmen oder elektrisch entwickelten Wärmen verwendet wird, werde ich vielleicht noch einmal zurückłommen. 
deren Differentiation partiell nach $a, a, b$ und $c$ liefert :

$$
\begin{aligned}
& \mathrm{ka}+\mathrm{b} \Sigma_{\mathrm{k}} \mathrm{t}+\mathrm{c} \Sigma_{\mathrm{k}} \mathrm{t}^{2}=\Sigma_{\mathrm{k}} q \text {; } \\
& \mathrm{l} a+\mathrm{b} \Sigma_{\mathrm{l}} \mathrm{t}+\mathrm{c} \Sigma_{\mathrm{t}} \mathrm{t}^{2}=\Sigma_{1} \mathrm{q} \text {; } \\
& a \Sigma_{k} t+a \Sigma_{1} t+b \Sigma_{k t} t^{2}+c \Sigma_{k} t^{3}=\Sigma_{k t} t q ; \\
& a \Sigma_{k} t^{2}+\alpha \Sigma_{1} t^{2}+b \Sigma_{k l} t^{3}+c \Sigma_{k l} t^{4}=\Sigma_{k l} t^{2} q \text {. }
\end{aligned}
$$

Die Elimination von $a$ aus den drei letzten Gleichungen liefert :

$$
\begin{aligned}
& \text { al } \Sigma_{k} t+b\left[1 \Sigma_{k 1} t^{2}-\left(\Sigma_{1} t\right)^{2}\right]+c\left[1 \Sigma_{k 1} t^{3}-\Sigma_{1} t \Sigma_{1} t^{2}\right] \\
& =1 \Sigma_{\mathrm{k}} \mathrm{t} \mathrm{q}-\Sigma_{\mathrm{i}} \mathrm{t} \Sigma_{\mathbf{l}} \mathrm{q} \\
& a \Sigma_{k} t^{3}+b\left[1 \Sigma_{k} t^{3}-\Sigma_{1} t \Sigma_{1} t^{2}\right]+c\left[1 \Sigma_{k} t^{4}-\left(\Sigma_{1} t^{2}\right)^{2}\right] \\
& =\mid \Sigma_{\mathrm{k} 1} \mathbf{t}^{2} \mathrm{q}-\Sigma_{\mathrm{l}} \mathbf{t}^{2} \Sigma_{\mathrm{l}} \mathbf{q} ;
\end{aligned}
$$

aus diesen beiden und der ersten der früheren Gleichungen kann $a, b$ und $c$ ohne weiteres bestimmt werden; ebenso wird $\alpha$ berochnet.

Viel einfacher gestaltet sich die Sache, wenn man $\mathfrak{c}=\mathbf{0}$ setzt, also annimmt, dafs die secundär eingesogene Quecksilbermenge der Zeit proportional ist, also durch die Formel a + bt dargestellt wird und der hineingeworfene Körper wieder blofs die Constante $a$ in $\alpha$ verwandelt, $b$ aber nicht àndert. Dann verwandelt sich die Gröfse, welche ein Minimum werden soll in :

$$
\Sigma_{k}(a+b t-q)^{2}+\Sigma_{1}(\alpha+b t-q)^{2} .
$$

denen Differentiation partiell nach a, $\alpha$ und $b$ liefert :

$$
\begin{aligned}
& \mathrm{ka}+\mathrm{b} \Sigma_{\mathrm{k}} \mathrm{t}=\boldsymbol{\Sigma}_{\mathrm{k}} \mathrm{q} \text {; } \\
& \mathrm{l} \alpha+\mathrm{b} \Sigma_{\mathrm{l}} \mathrm{t}=\Sigma_{\mathrm{l}} \mathrm{q} \text {; } \\
& a \Sigma_{k} t+a \Sigma_{1} t+b \dot{\Sigma}_{k \mid} t^{2}=\Sigma_{k l} t q ;
\end{aligned}
$$

woraus man ohne weiteres findet :

$$
\begin{aligned}
& \text { I. } \quad a=\frac{1 \Sigma_{k} t \Sigma_{k l} t q-\Sigma_{1} q \Sigma_{k} t \Sigma_{1} t-\mid \Sigma_{k} q \Sigma_{k l} t^{2}+\Sigma_{k} q\left(\Sigma_{l} t\right)^{2}}{1\left(\Sigma_{k} t\right)^{2}+k\left(\Sigma_{l} t\right)^{2}-k l \Sigma_{k l} t^{2}} \\
& \text { II. } \alpha=\frac{k \Sigma_{\mathrm{l}} \mathrm{t} \Sigma_{\mathrm{k} 1 \mathrm{q}} \mathrm{tq}-\Sigma_{\mathrm{k}} \Sigma_{\mathrm{l}} \mathrm{t} \Sigma_{\mathrm{k}} \mathrm{t}-\mathrm{k} \Sigma_{\mathrm{iq}} \Sigma_{\mathrm{kl}} \mathrm{t}^{2}+\Sigma_{\mathrm{l}} \mathrm{q}\left(\Sigma_{\mathrm{k}} \mathrm{t}\right)^{2}}{\mathrm{l}\left(\Sigma_{\mathrm{k}} \mathrm{t}\right)^{2}+\mathrm{k}\left(\Sigma_{1} \mathrm{t}\right)^{2}-\mathrm{kl} \Sigma_{\mathrm{k} !} \mathrm{t}^{2}} \\
& b=\frac{1 \Sigma_{k} q \Sigma_{k} t+k \Sigma_{1} t \Sigma_{1} q-k l \Sigma_{k 1} t q}{l\left(\Sigma_{k} t\right)^{2}+k\left(\Sigma_{1} t\right)^{2}-k l \Sigma_{k t} t^{2}}
\end{aligned}
$$

Dieselben Formeln erhält man, wenn man b erst aus den 
128 Boltzmann, Berechnung d. Calorimeterbeobachtungen.

Beobachtungen vor Einführung des Körpers, hierauf aus denen nach der Wärmeabgabe desselben in bekannter Weise nach der Methode der kleinsten Quadrate bestimmt, dann ersteren Werth mit k, letzteren mit 1 multiplicirt, die Producte addirt und die Summe durch $k+1$ dividirt. Die praktische Anwendung der letzten Formelu dürfe sich wohl hier und da lohnen; vielleicht auch, wo es sich un die gröfste Genauigkeit bandelt, die der ersteren, welche aber noch durch die in der Anmerkung besprochene Correction ergänzt werden mürsten.

Praktische Anwendung dor lotzten Formeln. - Der Beobachter lese vor dem Einwerfen des wärmeabgebenden Körpers in das Calorimeter $\mathrm{k}$ mal den Stand des Quecksilbers $q$ ab und notire dahei die Zeiten $t_{1}, t_{2} \ldots t_{k}$; ebenso nach muthmafslicher Vollendung der Wärmeabgabe $I \mathrm{mal}$ zu den Zeiten $t_{k+1}, t_{x+2}, \ldots t_{x+1}$. Aus diesen Beobachtungen berechne er $\Sigma_{k} t=t_{1}+t_{2}+\ldots+t_{k} ; \Sigma_{1} t=t_{k+1}$ $+t_{k+2}+\ldots+t_{k+1} ; \Sigma_{k 1} t q=t_{1} q_{1}+t_{2} q_{2}+\ldots$ $+t_{k} q_{k}+t_{k+1} q_{k+1}+\ldots+t_{k+1} q_{k+1}$ a. s. w. und setze die erhaltenen Werthe in die Gleichungen I und II ein. Aus a und $\alpha$ findet er dann die zu berechnende Warmemenge $\mathbf{Q}=a-\mathbf{a}$. 